\title{
EXISTENCE, UNIQUENESS, AND LONG-TIME BEHAVIOR OF MATERIALS WITH NONMONOTONE EQUATIONS OF STATE AND HIGHER-ORDER GRADIENTS
}

\author{
BY \\ K. KUTTLER AND E. C. AIFANTIS \\ Michigan Technological University, Houghton, Michigan
}

1. Introduction. Let the interval $[0,1]$ be occupied by a one-dimensional material of unit density. If the material is elastic, the stress is given by

$$
T=f(V),
$$

where $V_{x}$ is the strain. If elastic effects due to a material microstructure, such as a phase transformation or a continuous distribution of dislocations, are to be adequately included into the constitutive description, it has been noted by Aifantis and Serrin [2], among others, that an extra stress $T^{e}$ depending on long-range molecular forces should be added to (1.1). While there are many forms this extra stress could take, we will assume a particularly simple one involving the second spatial derivative of $V$. Then, the gradient-dependent expression for the total stress reads

$$
T=f(V)-\lambda V_{x x},
$$

where $f$ is a nonmonotone function and $\lambda$ a constant.

A central question is the determination of the stable equilibrium states associated with (1.2). One way to define these states is to consider (1.2) in conjunction with the differential equation of static equilibrium $T_{x}=0$ leading to the differential equation

$$
\left[f(V)-\lambda V_{x x}\right]_{x}=0
$$

which upon integration and for some constant $T_{0}$ implies

$$
f(V)-T_{0}-\lambda V_{x x}=0 .
$$

Alternatively, (1.4) can be obtained as the Euler-Lagrange equation for the functional

$$
I(V)=\int_{0}^{1}\left(F(V)+\frac{\lambda}{2} V_{x}^{2}\right) d x,
$$

where $F^{\prime}(V)=f(V)-T_{0}$. Gibbs's idea of stability is then to identify the stable states as those which yield local minima for this gradient-dependent energy functional.

A more fundamental and less restrictive way to define stability is to consider (1.2) in conjunction with the differential equation of motion or momentum balance 
$T_{x}=u_{t}$ where $u$ denotes the displacement $\left(V \equiv u_{x}\right)$. Then we can say that an equilibrium solution is stable if it is obtained as a long-time limit of the solution of the dynamical equations of motion for all initial data sufficiently close to the given equilibrium solution.

Obviously, there is a great deal more involved in this second definition than in the first. We must describe the equation of momentum balance or equation of motion along with a dynamic constitutive equation for the stress. This typically involves the inclusion of terms that give a viscous stress. Since these terms vanish at equilibrium, a given solution of (1.3) may be an equilibrium state of many different evolution equations. It does not seem obvious that the dynamic stability of this state should be the same for all these evolution equations. One must also address the problem of measuring closeness. There are many ways to measure this and it seems clear that it could happen that an equilibrium state is stable with respect to one measure of closeness and not stable for some other measure of closeness. It is not even obvious that solutions to the appropriate evolution equation exist or that they are unique. Because of these mathematical complexities, the idea of minimizing a functional has long been an attractive approach.

If this notion of stability could account for actual observations, perhaps there would be no need to study the second notion of stability with its increased mathematical complexities. This is not the case, however. Often, the material states of most interest today are states far from thermodynamic equilibrium (dissipative structures, metastable states), and no Lyapunov functional or minimization principle can be associated with them. Moreover, patterned solutions are usually ruled out by Gibbs's definition of stability as possessing more energy than other structured solutions. Patterned solutions, however, do occur as equilibrium solutions of (1.4). This was shown, for example, by Aifantis and Serrin [2] by a very simple argument. They showed that the bounded equilibrium solutions of equations including (1.4) as a special case are of three kinds: transitions, reversals, and oscillations. The oscillations correspond to the formation of patterns. The paper by Alexiades and Aifantis [1] suggests that in the sense of minimizing $I(V)$, the oscillations are unstable. However, their analysis was for the whole real line. Carr, Gurtin, and Slemrod [5] have shown by using a phase plane analysis that oscillations occur among the solutions of (1.4) on the finite interval if and only if $\lambda$ is sufficiently small. They also showed that these oscillations are unstable (in the sense of minimizing $(I(V))$. These results seem to imply that it is more appropriate to use the dynamical definition of stability.

The purpose of this paper is to discuss the existence, uniqueness, and long-time behavior of solutions of an initial boundary value problem, resulting from an equation of motion whose equilibrium states are solutions of (1.4). In Sec. 2, the evolution equation is derived. We note that a similar evolution equation was discussed, using different techniques, by Andrews and Ball [3], the difference being in the form of the viscous stress. We also allow for much more general boundary conditions. Section 3 contains an abstract form of the initial boundary value problem along with theorems of existence, uniqueness, and continuous dependence. Actually, this time-dependent problem should be called an "approximate problem" because various functions have 
been truncated. In Sec. 4, estimates on the strain are obtained which allow the determination of an approximate problem whose solution coincides with the solution of the problem of interest. Thus, well posedness of the transient problem is established. Also this section contains elegant estimates on the second and third derivatives of the strain. These estimates allow us to determine a space in which the trajectories of the transient problem are in a compact set. Moreover, if our method of proving existence and uniqueness is adapted to formulate a numerical method, these estimates would provide the necessary regularity to use standard approximation theory and thereby obtain error estimates for the numerical method. In Sec. 5 the long-time behavior of the solutions of the transient problem is partially obtained. We show that the velocity converges to 0 in $L^{2}(0,1)$ and for a given choice of initial data, we describe a set and a measure of distance such that the distance between this set and the solution to the transient problem converges to zero as $t \rightarrow \infty$. The ideas outlined here are essentially a version of standard techniques used in dynamical systems. In particular, Lemma 8 is a version of LaSalle's invariance principle. Finally, we show that for certain boundary conditions the transient solution converges to a solution of the steady-state problem. The overall approach of the paper is different from existing ones, since the present evolution equation cannot be viewed as a semilinear parabolic problem and thus treated by the techniques developed for these problems [8]. Nevertheless, our approach may be capable of being generalized to more nonlinear and less idealized equations.

Throughout the paper, $E$ is a closed subspace of $H^{2}(0,1)$ which contains the test functions, $C_{0}^{\infty}(0,1)$ and $H=L^{2}(0,1)$. Since $E$ is dense in $H$, we may write $E \subseteq H=H^{\prime} \subseteq E^{\prime}$ and we will always identify $H$ and $H^{\prime}$ in this way. The symbol $\rightarrow$ will denote strong convergence and $\rightarrow$ will denote weak or weak* convergence.

2. The evolution equation. Let $[0,1]$ be a material interval, $x \in[0,1]$, the initial density $\rho_{0}(x)=1$, and let $u$ be the displacement. Thus $u_{x}$ is the strain. We are interested in a stress given by

$$
T=f\left(u_{x}\right)-\lambda u_{x x x}+\text { viscous stress, }
$$

where $f$ is a nonmonotone function whose graph is given by Fig. 1.

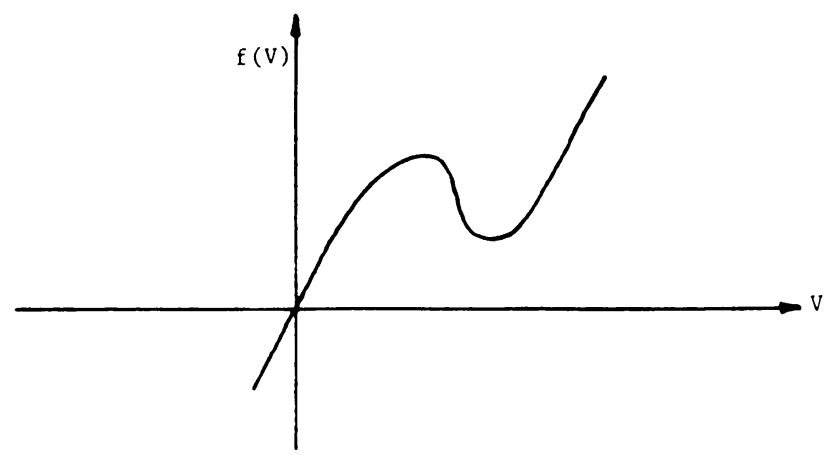

FIG. 1 . 
The viscous stress will be of the same general form as the elastic part of the stress, $f\left(u_{x}\right)-\lambda u_{x x x}$, but with a time derivative. Thus, the viscous stress is assumed to be of the general form

$$
\left(\beta\left(u_{x}\right)\right)_{t}-\delta u_{x x x t},
$$

with $\beta^{\prime}\left(u_{x}\right) \equiv \alpha\left(u_{x}\right)>0$ for all $u_{x}$. The term $u_{x x x t}$ is included in the viscous part of the stress because it seems reasonable to assume that if the gradient-dependent terms are important in the part of the steady-state or equilibrium stress, then the time derivative of these terms is also important in the transient or dynamical part of stress. The motion is then determined by the differential equation of balance of momentum $\left(T_{x}=u_{t t}\right)$, i.e.,

$$
\left.u_{t t}=\left(f\left(u_{x}\right)-\lambda u_{x x x}+\beta\left(u_{x}\right)\right) t-\delta u_{x x x t}\right)_{x}
$$

along with initial conditions

$$
u(0, x)=u_{0}, \quad u_{t}(0, x)=u_{1}(x),
$$

and boundary conditions, one of which will always be

$$
u(t, 1)=0 .
$$

This merely says that the right end of the material is fixed. For $\varphi \in C_{0}^{\infty}((0, T) \times$ $(0,1))$, multiply $(2.4)$ by $\varphi$ and integrate by parts. We have

$$
\begin{aligned}
& -\int_{0}^{T} \int_{0}^{1} u_{t}(t, x) \varphi_{t}(t, x) d x d t+\int_{0}^{T} \int_{0}^{1}\left(f\left(u_{x}\right)-P_{0}\right) \varphi_{x} d x d t \\
& \quad+\lambda \int_{0}^{T} \int_{0}^{1} u_{x x}(t, x) \varphi_{x x}(t, x) d x d t+\int_{0}^{T} \int_{0}^{1} \alpha\left(u_{x}\right) u_{x t} \varphi_{x} d x d t \\
& \quad+\delta \int_{0}^{T} \int_{0}^{1} u_{x x t} \varphi_{x x} d x d t=0
\end{aligned}
$$

where $P$ is a given constant. Let $E$ be a closed subspace of $H^{2}(0,1)$ containing $C_{0}^{\infty}(0,1)$. By requiring $(2.7)$ to hold for all $\varphi \in C_{0}^{\infty}(0, T ; E)$, we obtain a variational form for weak solutions to (2.4) and boundary conditions which are determined by choosing $E$. For example, if $E=\left\{u \in H^{2}(0,1): u(1)=0\right\}$, formal integration by parts in (2.7) yields the boundary conditions

$$
\begin{gathered}
u(t, 1)=0, \\
f\left(u_{x}(t, 0)\right)+\alpha\left(u_{x}(t, 0)\right) u_{x t}(t, 0)-\lambda u_{x x x}(t, 0)-\delta u_{x x x t}(t, 0)=P_{0}, \\
\lambda u_{x x}(t, 1)+\delta u_{x x t}(t, 1)=\lambda u_{x x}(t, 0)+\delta u_{x x x t}(t, 0)=0 .
\end{gathered}
$$

Other boundary conditions are obtained by routinely choosing $E \subseteq H^{2}(0,1)$.

In summary, the problem at hand is

$$
\begin{aligned}
& -\int_{0}^{T} \int_{0}^{1} u_{t}(t, x) \varphi_{t}(t, x) d x d t+\int_{0}^{T} \int_{0}^{1}\left(f\left(u_{x}\right)-P_{0}\right) \varphi_{x}+\int_{0}^{T} \int_{0}^{1} \lambda u_{x x} \varphi_{x x x} \\
& \quad+\int_{0}^{T} \int_{0}^{1} \alpha\left(u_{x}\right) u_{x t} \varphi_{x}+\delta \int_{0}^{T} \int_{0}^{1} u_{x x t} \varphi_{x x}=0
\end{aligned}
$$


for all $\varphi \in C_{0}^{\infty}(0, T ; E)$ where $C_{0}^{\infty}(0,1) \subseteq E \subseteq\left\{u \in H^{2}(0,1): u(1)=0\right\}$. The initial conditions take the form

$$
\begin{gathered}
v_{0} \in C(0, T ; E), \quad v_{1} \in C\left(0, T ; L^{2}(0,1)\right), \\
u(t, \cdot)=v_{0}(t) \text { a.e., } \quad u_{t}(t, \cdot)=v_{1}(t) \text { a.e. }, \\
\lim _{t \rightarrow 0+}\left\|u_{0}(t)-u_{0}\right\|_{E}+\left|v_{1}(t)-u_{1}\right|_{H}=0,
\end{gathered}
$$

where $H=L^{2}(0,1)$. Stated less precisely, this is

$$
\lim _{t \rightarrow 0+}\left\|u(t, \cdot)-u_{0}\right\|_{E}+\left|u_{t}(t, \cdot)-u_{1}\right|_{H}=0 .
$$

3. The abstract form of the evolution equation. For $u, v, w \in E$, it is natural to define the operators $Q(u), L$, and $R$ mapping $E$ to $E^{\prime}$ by

$$
\begin{gathered}
\langle Q(u) v, w\rangle=\left(\alpha_{r}\left(u_{x}\right) v_{x}, w_{x}\right)_{H}, \\
\langle L u, w\rangle=\left(u_{x x}, w_{x x}\right)_{H}, \\
\langle R u, v\rangle=\lambda\langle L u, v\rangle+\left(f_{r}\left(u_{x}\right)-P_{0}, v_{x}\right)_{H},
\end{gathered}
$$

where for $h \in\{\alpha, f\}$,

$$
h_{r}(x)= \begin{cases}h(x) & \text { if }|x|<r, \\ h(r) & \text { if } x \geq r, \\ h(-r) & \text { if } x \leq-r,\end{cases}
$$

with $f$ assumed to be locally Lipschitz and $\alpha$ continuous. The abstract form of (2.9) $)_{r}\left[\left(\right.\right.$ problem (2.9) with $f$ replaced by $f_{r}$ and $\alpha$ replaced by $\left.\left.\alpha_{r}\right)\right]$ to begin with is

$$
\begin{gathered}
u^{\prime \prime}+Q(u) u^{\prime}+\delta L u^{\prime}+R u=0, \\
u^{\prime}, u \in L^{2}(0, T ; E) \equiv \mathscr{V}, \quad u^{\prime \prime} \in \mathscr{V}^{\prime}, \\
u(0)=u_{0} \in E, \quad u^{\prime}(0)=u_{1} \in H,
\end{gathered}
$$

where the "'" denotes differentiation in the sense of $E^{\prime}$ valued distributions. That is, for $g \in L^{1}\left(0, T ; E^{\prime}\right)$, and $\varphi \in C_{0}^{\infty}(0, T)$,

$$
g^{\prime}(\varphi) \equiv-\int_{0}^{T} g(t) \varphi^{\prime}(t) d t
$$

THEOREM 1. There exists a unique solution to (3.3).

The proof of Theorem 1 is a routine modification of the proofs of similar theorems found in $[11,9,10]$, so we omit it.

Now define $M: E \rightarrow E^{\prime}$ by

$$
\langle M u, v\rangle=\left(\beta_{r}\left(u_{x}\right)-\beta_{r}\left(u_{0 x}\right), v_{x}\right)_{H}+\delta\left(u_{x x}-u_{0 x x}, v_{x x}\right)_{H}
$$

where

$$
\beta_{r}(V)=\int_{0}^{V} \alpha_{r}(s) d s
$$


Applying $\int_{0}^{t}$ to both sides of $(2.3 .1)$ we obtain

$$
\begin{gathered}
u^{\prime}(t)+M u(t)+\int_{0}^{t} R u(s) d s=u_{1}, \\
u(0)=u_{0}, \\
u, u^{\prime} \in \mathscr{V} .
\end{gathered}
$$

LEMMA 1. $u$ solves (3.3) if and only if $u$ solves (3.7).

THEOREM 2. Let $u_{0 n} \in E$ and $u_{1 n} \in H$ be given sequences of initial data satisfying

$$
\lim _{n \rightarrow \infty}\left(\left\|u_{0 n}-u_{0}\right\|_{E}+\left|u_{1 n}-u_{1}\right|_{H}\right)=0 .
$$

Then if $u_{n}$ is the solution of $(3.3)_{n}$, i.e., the problem (3.3) with $u_{0 n}$ and $u_{1 n}$ in place of $u_{0}$ and $u_{1}$, respectively, we have

$$
\lim _{t \rightarrow \infty}\left(\sup _{t \in[0, T]}\left\|u_{n}(t)-u(t)\right\|_{E}\right)=0 .
$$

Proof. From (3.7) it is easy to obtain

$$
\begin{aligned}
& \frac{1}{2}\left|u_{n}(t)-u(t)\right|_{H}^{2}-\frac{1}{2}\left|u_{0 n}-u_{0}\right|_{H}^{2}+\int_{0}^{t}\left\langle M u_{n}(s)-M u(s), u_{n}(s)-u(s)\right\rangle d s \\
& \quad+\int_{0}^{t} \int_{0}^{s}\left\langle R u_{n}(y)-R u(y), u_{n}(s)-u(s)\right\rangle d y d s=\int_{0}^{t}\left(u_{1 n}-u_{1}, u_{n}(s)-u(s)\right) d s .
\end{aligned}
$$

Consider the fourth term. This term is bounded in absolute value by

$$
K_{r} \int_{0}^{t} \sqrt{s}\left(\int_{0}^{s}\left\|u_{n}(y)-u(y)\right\|_{E}^{2} d y\right)^{1 / 2}\left\|u_{n}(s)-u(s)\right\|_{E} d s
$$

where $K_{r}$ is a constant depending only on $r$. It follows that this may be dominated by an expression of the form

$$
\int_{0}^{t}\left[C_{r, \eta} s \int_{0}^{s}\left\|u_{n}(y)-u(y)\right\|_{E}^{2} d y+\eta\left\|u_{n}(s)-u(s)\right\|_{E}^{2}\right] d s,
$$

where $\eta>0$ is arbitrary and $C_{r, \eta}$ depends on $r$ and $\eta$. The third term of (3.10) is easily seen to be no smaller than

$$
\int_{0}^{l} \theta_{r}\left(\| u_{n}(s)-\left.u(s)\right|_{E} ^{2}-\left|u_{n}(s)-u(s)\right|_{H}^{2}\right) d s,
$$

for some constant $\theta_{r}>0$ depending on $r$. It follows that there exists a constant $K_{r}$ depending on $r$ such that

$$
\begin{aligned}
\mid u_{n}(t) & -\left.u(t)\right|_{H} ^{2}+\int_{0}^{t} \| u_{n}(s)-\left.u(s)\right|_{E} ^{2} d s \\
\leq & K_{r}\left(\left|u_{1 n}-u_{1}\right|_{H}^{2}+\left|u_{0 n}-u_{0}\right|_{H}^{2}\right) \\
& +K_{r} \int_{0}^{t}(1+s)\left(\int_{0}^{s} \| u_{n}(y)-\left.u(y)\right|_{E} ^{2} d y+\left|u_{n}(s)-u(s)\right|_{H}^{2}\right) d s .
\end{aligned}
$$


By Gronwall's lemma,

$$
\left|u_{n}(t)-u(t)\right|_{H}^{2}+\int_{0}^{t}\left\|u_{n}(s)-u(s)\right\|_{E}^{2} d s \leq K_{r}\left(\left|u_{1 n}-u_{1}\right|_{H}^{2}+\left|u_{0 n}-u_{0}\right|_{H}^{2}\right) e^{T+T^{2} / 2}
$$

Therefore, $\lim _{t \rightarrow \infty}\left|u_{n}(t)-u(t)\right|_{H}=0$ and $\lim _{n \rightarrow \infty}\left\|u_{n}-u\right\|_{\mathcal{Y}}=0$. Multiply (3.3.1) by $u_{n}^{\prime}$ and then apply $\int_{0}^{t}$. Since $f_{r}$ is bounded this yields

$$
\begin{aligned}
& \left|u_{n}^{\prime}(t)\right|_{H}^{2}+\theta_{r} \int_{0}^{t}\left(\left|u_{n x}^{\prime}(s)\right|_{H}^{2}+\left|u_{n x x}^{\prime}(s)\right|_{H}^{2}\right) d s+\lambda\left|u_{n x x}(t)\right|_{H}^{2} \\
& \quad \leq \lambda\left|u_{0 n x x}\right|_{H}^{2}+K_{r} \int_{0}^{t}\left|u_{n x}^{\prime}(s)\right|_{H} d s,
\end{aligned}
$$

where $\theta_{r}$ and $K_{r}$ are positive constants depending on $r$. The above inequality implies easily the existence of a constant, $C$, depending on $r$ but independent of $n$ such that

$$
\left\|u^{\prime}\right\|_{y}, \quad\left\|u_{n}^{\prime}\right\|_{y} \leq C .
$$

Because of (3.17), we may take $u_{n}, u$ to be in $C(0, T ; E)$.

Lemma 2. For each $t \in[0, T], \lim _{n \rightarrow \infty} u_{n}(t)=u(t)$.

Proof. Suppose $u_{n}\left(t_{0}\right) \nrightarrow u\left(t_{0}\right)$. Then by selecting a subsequence, still denoted by $n$, we may assume

$$
\begin{aligned}
& \left\|u_{n}(t)-u(t)\right\|_{E} \rightarrow 0 \text { a.e.t } \\
& \left\|u_{n}\left(t_{0}\right)-u\left(t_{0}\right)\right\|_{E} \geq \varepsilon>0 .
\end{aligned}
$$

But then

$$
\begin{aligned}
\left\|u_{n}\left(t_{0}\right)-u\left(t_{0}\right)\right\| & \leq\left\|u_{n}\left(t_{0}\right)-u_{n}(t)\right\|+\left\|u_{n}(t)-u(t)\right\|+\left\|u(t)-u\left(t_{0}\right)\right\| \\
& \leq 2 C\left|t_{0}-t\right|^{1 / 2}+\left\|u_{n}(t)-u(t)\right\| .
\end{aligned}
$$

Let $t$ be such that $\left\|u_{n}(t)-u(t)\right\|_{E} \rightarrow 0$ and $2 C\left|t_{0}-t\right|^{1 / 2}<\frac{\varepsilon}{2}$. Letting $n \rightarrow \infty$ in (3.19) contradicts (3.18.2). This proves Lemma 2. To complete the proof of Theorem 2, let $\varepsilon>0$ be given and let $0=t_{0}<t_{1}<\cdots<t_{m}=T$ be a uniform partition with $2\left(t_{i}-t_{i-1}\right)^{1 / 2} C<\frac{\varepsilon}{2}$. Let $n_{0}$ be large enough such that for $n \geq n_{0}$, $\left\|u_{n}\left(t_{i}\right)-u\left(t_{i}\right)\right\|_{E}<\frac{\varepsilon}{2}$ for $i=0, \ldots, m$. Then for any $t \in(0, T], t \in\left(t_{i-1}, t_{i}\right]$ for some $i$ and thus for any $n \geq n_{0}$,

$$
\left\|u_{n}(t)-u(t)\right\| \leq 2 C\left|t_{i}-t\right|^{1 / 2}+\left\|u_{n}\left(t_{i}\right)-u\left(t_{i}\right)\right\|<\frac{\varepsilon}{2}+\frac{\varepsilon}{2}=\varepsilon .
$$

This proves Theorem 2 .

4. Estimates. In the remainder of the paper, we assume that there exists an $r_{0}>0$ such that

$$
\begin{gathered}
\left|u_{0 x}(x)\right|<r_{0}, \\
f(V)-P_{0}>0 \quad \text { if } V>r_{0}, \\
f(V)-P_{0}<0 \quad \text { if } V<-r_{0} .
\end{gathered}
$$


Define

$$
W(V)=\int_{0}^{V}\left(f(s)-P_{0}\right) d s, \quad W_{r}(V)=\int_{0}^{V}\left(f_{r}(s)-P_{0}\right) d s,
$$

and assume

$$
\lim _{V \rightarrow-\infty} W(V)=\lim _{V \rightarrow \infty} W(V)=\infty .
$$

From now on, assume $r>r_{0}$ and let

$$
-J=\min \{W(V): V \in \mathbb{R}\} .
$$

It follows, since $r>r_{0}$, that

$$
-J=\min \left\{W_{r}(V): V \in \mathbb{R}\right\} .
$$

Multiply (3.3.1) by $u^{\prime}$ and integrate $\int_{0}^{t}$. This yields

$$
\begin{gathered}
\frac{1}{2}\left|u^{\prime}(t)\right|_{H}^{2}-\frac{1}{2}\left|u_{1}\right|_{H}^{2}+\int_{0}^{t}\left\langle R u(s), u^{\prime}(s)\right\rangle d s \\
+\int_{0}^{t}\left(\alpha_{r}\left(u_{x}(s)\right) u_{x}^{\prime}(s), u_{x}^{\prime}(s)\right)_{H} d s \\
+\delta \int_{0}^{t}\left|u_{x x}^{\prime}(s)\right|_{H}^{2} d s=0 .
\end{gathered}
$$

Consider the third term. From (4.2.1) and (3.1.3),

$$
\begin{aligned}
\int_{0}^{t}\left\langle R u(s), u^{\prime}(s)\right\rangle d s= & \frac{\lambda}{2}\left|u_{x x}(t)\right|_{H}^{2}-\frac{\lambda}{2}\left|u_{0 x x}\right|_{H}^{2} \\
& +\int_{0}^{1} W_{r}\left(u_{x}(t)(x)\right) d x-\int_{0}^{1} W\left(u_{0 x}(x)\right) d x .
\end{aligned}
$$

It follows from (4.3) that

$$
\begin{aligned}
\lambda \mid u_{x x} & \left.(t)\right|_{H} ^{2}+\int_{0}^{1} W_{r}\left(u_{x}(t)(x)\right) d x \\
& \leq \frac{1}{2}\left|u_{1}\right|_{H}^{2}+\lambda\left|u_{0 x x}\right|_{H}^{2}+\int_{0}^{1} W\left(u_{0 x}(x) d x\right. \\
& =C,
\end{aligned}
$$

where $C$ is a constant independent of $r, t, \beta$, and $\delta$. For simplicity in notation, let $V(x)=u_{x}(t)(x)$. Then (4.6) becomes

$$
\lambda\left|V_{x}(x)\right|_{H}^{2}+\int_{0}^{1} W_{r}(V(x)) d x \leq C .
$$

Therefore

$$
\left|V_{x}(x)\right|_{H}^{2} \leq(C+J) \lambda^{-1} \equiv C_{1}^{2},
$$

and so for $x, y \in[0,1]$

$$
|V(x)-V(y)| \leq C_{1}|x-y|^{1 / 2} \leq C_{1} .
$$


Let $r_{1}>r_{0}$ and let $r_{1}$ satisfy

$$
W(V)>C \quad \text { if }|V| \geq r_{1} .
$$

Hereafter let $r>r_{1}$. Then $W_{r}(V)=W(V)$ if $|V|<r$ and there exists $x_{0} \in[0,1]$ such that $\left|V\left(x_{0}\right)\right|<r_{1}$. If this were not so, then the second term in (4.7) would be larger than $C$. Therefore, for any $x \in[0,1]$,

$$
\begin{aligned}
|V(x)| & \leq\left|V\left(x_{0}\right)\right|+C_{1} \\
& \leq C_{1}+r_{1} \equiv C_{2} .
\end{aligned}
$$

This proves

Lemma 3. If $r>r_{1}$, there exists a constant $C_{2}$, independent of $t, r, \delta$, and $\beta$ such that

for all $t>0$ and $x \in[0,1]$.

$$
\left|u_{x}(t)(x)\right| \leq C_{2}
$$

For the remainder of the paper, $r>\max \left(r_{1}, C_{2}\right) \equiv r_{2}$. Then, $\left|u_{x}(t)(x)\right|<r_{2}<r$ and thus, a measurable representative of the problem (3.3) is the solution to problem (2.9) which satisfies

$$
u, u_{t}, u_{t x}, u_{t x x}, u_{x}, u_{x x} \in L^{2}((0, T) \times(0,1)) .
$$

Since $T>0$ is arbitrary, Lemma 3 along with Theorems 1 and 2 establish global existence, uniqueness, and continuous dependence for problems (2.9) and (4.13). Furthermore, we can study this problem in the form (3.3) or (3.7) for a suitable choice of $r>0$, the choice of $r$ being independent of the function $\beta$, the constant, $\delta$, and $t$.

Up to now, the subspace $E \subseteq H^{2}(0,1)$ is arbitrary as long as $C_{0}^{\infty}(0,1) \subseteq E \subseteq$ $\left\{u \in H^{2}(0,1): u(1)=0\right\}$. We now assume that whenever $g \in C_{0}^{\infty}(0,1)$,

$$
\int_{1}^{(\cdot)} g(s) d s \in E
$$

and

$$
u_{0} \in E \cap H^{3}(0,1) \text {. }
$$

Of course $u_{0}=0$ satisfies (4.14.2) and (4.14.1) does not seem to be a serious restriction either.

Let $g \in C_{0}^{\infty}(0,1)$ be given. Then multiply (3.7.1) by the function given by $x \rightarrow \int_{1}^{x} g(z) d z$. This yields

$$
\begin{aligned}
& \int_{0}^{1} u^{\prime}(t)(x)\left(\int_{1}^{x} g(z) d z\right) d x \\
& \quad+\int_{0}^{t}\left(\int_{0}^{1} \lambda u_{x x}(s)(x) g_{x}(x)+\left(f_{r}\left(u_{x}(s)(x)\right)-P_{0}\right) g(x) d x\right) d s \\
& +\int_{0}^{1}\left[\beta_{r}\left(u_{x}(t)(x)\right)-\beta_{r}\left(u_{0 x}(x)\right)\right] g(x)+\delta\left(u_{x x}(t)(x)-u_{0 x x}(x)\right) g_{x}(x) d x \\
& \quad=\int_{0}^{1} u_{1}(x)\left(\int_{1}^{x} g(z) d z\right) d x .
\end{aligned}
$$


Let

$$
\begin{aligned}
q(t)= & \int_{0}^{1} u^{\prime}(t)(x)\left(\int_{1}^{x} g(z) d z\right) d x+\int_{0}^{1}\left[\beta_{r}\left(u_{x}(t)(x)\right)-\beta_{r}\left(u_{0 x}(x)\right)\right] g(x) d x \\
& +\delta \int_{0}^{1}\left[u_{x x}(t)(x)-u_{0 x x}(x)\right] g_{x}(x) d x-\int_{0}^{1} u_{1}(x)\left(\int_{1}^{x} g(z) d z\right) d x .
\end{aligned}
$$

From (4.15) (note $q(t)$ consists of the terms of (4.15) that do not have $\int_{0}^{t}$ next to them) we obtain

$$
q^{\prime}(t)=-\lambda \int_{0}^{1} u_{x x}(t)(x) g_{x}(x) d x-\int_{0}^{1}\left[f_{r}\left(u_{x}(t)(x)\right)-P_{0}\right] g(x) d x .
$$

Therefore,

$$
\begin{aligned}
q^{\prime}(t)+\frac{\lambda}{\delta} q(t)= & \frac{\lambda}{\delta} \int_{0}^{1} u^{\prime}(t)(x)\left(\int_{1}^{x} g(z) d z\right) d x \\
& +\frac{\lambda}{\delta} \int_{0}^{1}\left[\beta_{r}\left(u_{x}(t)(x)\right)-\beta_{r}\left(u_{0 x}(x)\right)\right] g(x) d x \\
& +\frac{\lambda}{\delta} \int_{0}^{1} u_{0 x x x}(x) g(x)-\frac{\lambda}{\delta} \int_{0}^{1} u_{1}(x)\left(\int_{1}^{x} g(z) d z\right) d x \\
& -\int_{0}^{1}\left[f_{r}\left(u_{x}(t)(x)\right)-P_{0}\right] g(x) d x .
\end{aligned}
$$

Next, we assume $|g|_{H} \leq 1$. From (4.5) and (4.4) it follows that $\left|u^{\prime}(t)\right|_{H}$ is bounded independent of $r, \delta$, and $t$. Therefore, from Lemma 3 and (4.18), there exists a constant $C$, independent of $r, \delta, t$, and $g \in C_{0}^{\infty}(0,1)$ with $|g|_{H} \leq 1$ such that

$$
q^{\prime}(t)+\frac{\lambda}{\delta} q(t) \leq \frac{C}{\delta}+C \equiv C_{1} .
$$

From (4.16) and (3.7.3), $q(0)=0$. Therefore

$$
q(t) \leq C_{1}\left[\frac{\delta}{\lambda}-\frac{\delta}{\lambda} e^{(\lambda t / \delta)}\right] \leq \frac{C_{1} \delta}{\lambda} .
$$

This along with (4.16) and Lemma 3 implies that for all $g \in C_{0}^{\infty}(0,1)$ with $|g|_{H} \leq$ 1 , there is a constant $C_{3}$ independent of $r, t$, such that

$$
\delta \int_{0}^{1} u_{x x}(t)(x) g_{x}(x) d x \leq C_{3} .
$$

Since this holds for all $g \in C_{0}^{\infty}(0,1)$ with $|g|_{H} \leq 1$, it follows that

$$
\sup _{\substack{|g|_{H} \leq 1 \\ g \in C_{0}^{\infty}(0,1)}}\left|\int_{0}^{1} u_{x x}(t)(x) g_{x} d x\right| \leq \frac{C_{3}}{\delta}
$$

It follows from the density of $C_{0}^{\infty}(0,1)$ in $H=L^{2}(0,1)$, that there exists a unique function $k \in H$ such that $(k, g)_{H}=u_{x x x}(t)(g)$. Thus

$$
u_{x x x}(t) \in H \quad \text { and } \quad\left|u_{x x x}(t)\right|_{H} \leq \frac{C_{3}}{\delta}
$$


for all $t>0$. This establishes the following

Lemma 4. Let $u_{0} \in E \cap H^{3}(0,1)$ and let (4.14.1) hold for all $g \in C_{0}^{\infty}(0,1)$. Then there exists $C$, independent of $t$ and $r$, such that

$$
\left|u_{x x x}(t)\right|_{H} \leq C .
$$

Corollary 1. Let $u_{0} \in E \cap H^{4}(0,1)$ and let (4.14.1) hold for all $g \in C_{0}^{\infty}(0,1)$. Then there exists a constant $C$, independent of $t$ and $r$, such that

$$
\left|u_{x x x x}(t)\right|_{H} \leq C \text {. }
$$

Proof. Replace $g$ by $g_{x}$ in (4.15), (4.16), (4.17). This yields

$$
\begin{aligned}
q(t)= & \int_{0}^{1} u^{\prime}(t)(x) g(x) d x-\int_{0}^{1}\left(\beta^{\prime}\left(u_{x}\right) u_{x x}-\beta^{\prime}\left(u_{0 x}\right) u_{0 x x}\right) g(x) d x \\
& +\delta \int_{0}^{1} u_{x x}(t)(x) g_{x x}(x) d x-\delta \int_{0}^{1} u_{0 x x x x}(x) g(x) d x-\int_{0}^{1} u_{1}(x) g(x) d x \\
q^{\prime}(t)= & -\lambda \int_{0}^{1} u_{x x}(t)(x) g_{x x}(x) d x+\int_{0}^{1} f^{\prime}\left(u_{x}\right) u_{x x}(t)(x) g(x) d x
\end{aligned}
$$

Thus

$$
\begin{aligned}
q^{\prime}(t)+\frac{\lambda}{\delta} q(t)= & \frac{\lambda}{\delta} \int_{0}^{1} u^{\prime}(t)(x) g(x) d x \\
& -\frac{\lambda}{\delta} \int_{0}^{1}\left(\beta^{\prime}\left(u_{x}\right) u_{x x}(t)(x)-\beta^{\prime}\left(u_{0 x}\right) u_{0 x x}(x)\right) g(x) d x \\
& -\lambda \int_{0}^{1} u_{0 x x x x}(x) g(x) d x-\frac{\lambda}{\delta} \int_{0}^{1} u_{1}(x) g(x) d x \\
& +\int_{0}^{1} f^{\prime}\left(u_{x}\right) u_{x x}(t)(x) g(x) d x .
\end{aligned}
$$

Let $C$ be a constant independent of $r$ and $t$. In the same way as before, $q^{\prime}(t)+$ $\frac{\lambda}{\delta} q(t) \leq C$ where $C$ is independent of $t, r$, and $g \in C_{0}^{\infty}(0,1)$ with $|g|_{H} \leq 1$. Thus $q(t) \leq C$ and it follows that for all $g \in C_{0}^{\infty}(0,1)$ with $|g|_{H} \leq 1$,

$$
\left|\int_{0}^{1} u_{x x}(t)(x) g_{x x}(x) d x\right| \leq C .
$$

Thus $\left|u_{x x x x}(t)(g)\right| \leq C$, so by the density of $C_{0}^{\infty}(0,1)$ in $L^{2}(0,1)$ and the Riez representation theorem, $u_{x x x x x}(t) \in L^{2}(0,1)$ and $\left|u_{x x x x}(t)\right|_{H} \leq C$.

5. The long-time behavior. Let us define

$$
\begin{gathered}
B=\left\{u \in E:\left|u_{x}(x)\right|<r \text { for some } r>0 \text { and } u_{x x x} \in H\right\}, \\
F=H^{3}(0,1) \cap E .
\end{gathered}
$$

Then $B \subseteq F$. The content of Sec. 2-4 imply that problem (3.3) yields a dynamical system on $B \times H$ given by

$$
S(t)\left(u_{0}, u_{1}\right)=\left(u(t), u^{\prime}(t)\right)
$$


where $u$ is the solution of problem (3.3) with initial data $\left(u_{0}, u_{1}\right) \in B \times H$. By this we mean

$$
S\left(t_{1}+t_{2}\right)\left(u_{0}, u_{1}\right)=S\left(t_{1}\right) S\left(t_{2}\right)\left(u_{0}, u_{1}\right),
$$

which follows from the uniqueness of problem (3.3).

Lemma 5. Let $u$ be the solution of problem (3.3). Then

$$
\lim _{t \rightarrow \infty}\left|u^{\prime}(t)\right|_{H}=0 \text {. }
$$

Proof. Applying $\frac{d}{d t}$ to both sides of (4.4) and using the estimates of Sec. 4 for $u_{x}(t)(x)$, we obtain an inequality of the form

$$
\left.\frac{d}{d t}\left|u^{\prime}(t)\right|_{H}^{2}-K\left(\left|u_{x}^{\prime}(t)\right|_{H}+\left|u_{x x}^{\prime}(t)\right|\right)+\left.C\left(\left|u_{x}^{\prime}(t)\right|_{H}^{2}+\mid u_{x x}^{\prime}(t)\right)\right|_{H} ^{2}\right) \leq 0
$$

where $K$ and $C$ are two constants which are independent of $t$. It follows from this that $\frac{d}{d t}\left|u^{\prime}(t)\right|_{H}^{2}$ is bounded above. The estimate for $u_{x}(t)(x)$ of Sec. 4 applied to the fourth term of (4.4) along with (4.4) and (4.5) yields

$$
\int_{0}^{t}\left|u_{x}^{\prime}(s)\right|_{H}^{2} d s \leq C
$$

where $C$ is a constant independent of $t$. Poincaré's inequality (recall $E \subseteq\{u \in$ $\left.\left.H^{2}(0,1): u(1)=0\right\}\right)$ yields

$$
\int_{0}^{t}\left|u^{\prime}(s)\right|_{H}^{2} d s \leq C
$$

This, with the upper bound on $\frac{d}{d t}\left(\left|u^{\prime}(t)\right|_{H}^{2}\right)$ implies the desired conclusion.

Next, we assume $\left(u_{0}, u_{1}\right) \in B \times H$.

LemMA 6. For each $\left(u_{0}, u_{1}\right) \in B \times H$, there exists $\left(y_{0}, y_{1}\right) \in B \times H$ and a sequence $t_{n} \rightarrow \infty$ such that $S\left(t_{n}\right)\left(u_{0}, u_{1}\right)-\left(y_{0}, y_{1}\right)$ in $F \times H$. Furthermore, $y_{1}=0$.

Proof. This follows from Lemma 5 and the estimates of Sec. 4.

Define the $\omega$ limit set of a point $\left(u_{0}, u_{1}\right) \in B \times H$ by

$$
\omega\left(u_{0}, u_{1}\right)=\bigcap_{t_{0}>0} \text { weak closure }\left\{S(t)\left(u_{0}, u_{1}\right): t \geq t_{0}\right\} .
$$

Lemma 6 implies that $\omega\left(u_{0}, u_{1}\right) \neq \varnothing$ and Lemma 5 implies that all elements of $\omega\left(u_{0}, u_{1}\right)$ are of the form $\left(y_{0}, 0\right)$. For $(u, v) \in B \times H$, define

$$
G(u, v)=\frac{1}{2}|v|_{H}^{2}+\int_{0}^{1} W\left(u_{x}(x)\right) d x+\frac{\lambda}{2}\left|u_{x x}\right|_{H}^{2} .
$$

From (4.4) and (4.5) we have

$$
G\left(S(t)\left(u_{0}, u_{1}\right)\right)+\int_{0}^{t}\left\langle Q(u) u^{\prime}, u^{\prime}\right\rangle+\delta\left\langle L u^{\prime}, u^{\prime}\right\rangle d s=G\left(u_{0}, u_{1}\right) .
$$

The integrand in (5.10) is nonnegative; thus it follows that $G\left(S(t)\left(u_{0}, u_{1}\right)\right)$ is decreasing in $t$ and thus $G$ is a Lyapunov function because it decreases along trajectories. Also,

$$
G\left(S(t)\left(u_{0}, u_{1}\right)\right) \geq-J,
$$


where $-J$ is the lower bound to $W$. Let

$$
l\left(u_{0}, u_{1}\right)=\lim _{t \rightarrow \infty} G\left(S(t)\left(u_{0}, u_{1}\right)\right) .
$$

LemMa 7. For $\left(y_{0}, 0\right) \in \omega\left(u_{0}, u_{1}\right), G\left(y_{0}, 0\right)=l\left(u_{0}, u_{1}\right)$.

Proof. If $u$ is the solution of (3.3) with $\left(u_{0}, u_{1}\right) \in B \times H$, there exists $t_{n} \rightarrow \infty$ such that $u\left(t_{n}\right) \rightarrow y_{0}$ in $F$. By the compactness of the embedding of $H^{3}(0,1)$ into $H^{2}(0,1)$ and Lemma 5, this implies $u\left(t_{n}\right) \rightarrow y_{0}$ in $E$ and $u^{\prime}\left(t_{n}\right) \rightarrow 0$ in $H$. Therefore, $l\left(u_{0}, u_{1}\right)=\lim _{n \rightarrow \infty} G\left(u\left(t_{n}\right), u^{\prime}\left(t_{n}\right)\right)=G\left(y_{0}, 0\right)$.

LEMMA 8. Let $Z$ be the set of equilibrium solutions of (3.3.1) which are in $B$. That is, $u \in Z$ if and only if

$$
R u=0, \quad u \in B
$$

Then

$$
\omega\left(u_{0}, u_{1}\right) \subseteq Z \times\{0\} .
$$

Proof. Let $\left(y_{0}, 0\right) \in \omega\left(u_{0}, u_{1}\right)$ and consider $G\left(S(\tau)\left(y_{0}, 0\right)\right)$.

$$
\left.G\left(S(\tau) y_{0}, 0\right)\right) \leq G\left(y_{0}, 0\right)=l\left(u_{0}, u_{1}\right) .
$$

Let $t_{n} \rightarrow \infty$ and $S\left(t_{n}\right)\left(u_{0}, u_{1}\right) \rightarrow\left(y_{0}, 0\right)$ in $F \times H$. By Lemma 5 and the compactness of the embedding of $H^{3}(0,1)$ into $H^{2}(0,1)$, this means $S\left(t_{n}\right)\left(u_{0}, u_{1}\right) \rightarrow$ $\left(y_{0}, 0\right)$ in $E \times H$. Thus, by Theorem 2,

$$
S\left(\tau+t_{n}\right)\left(u_{0}, u_{1}\right)=S(\tau) S\left(t_{n}\right)\left(u_{0}, u_{1}\right) \rightarrow S(\tau)\left(y_{0}, 0\right),
$$

the convergence taking place in $E \times H$. This implies

$$
l\left(u_{0}, u_{1}\right)=\lim _{n \rightarrow \infty} G\left(S\left(\tau+t_{n}\right)\left(u_{0}, u_{1}\right)\right)=G\left(S(\tau)\left(y_{0}, 0\right)\right) .
$$

This shows that $t \rightarrow G\left(S(t)\left(y_{0}, 0\right)\right)$ is constant and so from $(5.10), S(t)\left(y_{0}, 0\right)=$ $\left(y_{0}, 0\right)$ which implies $\left(y_{0}, 0\right)$ is in $Z \times\{0\}$.

Let $A$ be a subset of $B$ and for $v \in E$, define

$$
\operatorname{dist}(v, A)=\inf \left\{\|v-y\|_{E}: y \in A\right\} .
$$

THeOrem 3. For $\left(u_{0}, u_{1}\right) \in B \times H$, let $\pi_{1}\left(u_{0}, u_{1}\right)=u_{0}$. Then

$$
\lim _{t \rightarrow \infty} \operatorname{dist}\left(u(t), \pi_{1}\left(\omega\left(u_{0}, u_{1}\right)\right)\right)=0 .
$$

Proof. If (5.19) is not true, there exist $\varepsilon>0$ and a sequence $t_{n} \rightarrow \infty$ such that $\operatorname{dist}\left(u\left(t_{n}\right), \pi_{1}\left(\omega\left(u_{0}, u_{1}\right)\right)\right) \geq \varepsilon$. But from Sec. 4, there is a subsequence $t_{n^{\prime}} \rightarrow \infty$ such that $u\left(t_{n^{\prime}}\right)-y_{0}$ in $F$ for some $y_{0} \in B$. By Lemma 5, $\left(y_{0}, 0\right) \in \omega\left(u_{0}, u_{1}\right)$. By the compactness of the embedding of $F$ into $E, \lim _{n^{\prime} \rightarrow \infty}\left\|u\left(t_{n^{\prime}}\right)-y_{0}\right\|_{E}=0$. i.e., a contradiction.

Corollary 2. In the situation of Theorem $3, \lim _{t \rightarrow \infty} \operatorname{dist}(u(t), Z)=0$. The proof follows from Lemma 8 . For more background on the procedure just presented, we refer to $[4,6,7]$.

In fact, the conclusion of Theorem 3 can be strengthened if we assume that $E=$ $\left\{u \in H^{2}(0,1): u(1)=0\right\}$, implying that the natural boundary conditions (2.8.3) 
and (2.8.2) are satisfied. For the remainder of this paper, we assume this is the case. If $(z, 0) \in \omega\left(u_{0}, u_{1}\right)$ where $\left(u_{0}, u_{1}\right) \in B \times H$, it follows from Lemma 8 that $R z=0, z \in B$. Therefore,

$$
\lambda z_{x x x x}-\left(f\left(z_{x}\right)-P_{0}\right)_{x}=0,
$$

and

$$
z_{x x}(1)=z_{x x}(0)=0, \quad z \in H^{4}(0,1) .
$$

Letting $V=z_{x}$, it follows from the choice of $E$ that

$$
\begin{gathered}
-\lambda V_{x x}+f(V)-P_{0}=0, \\
V_{x}(1)=V_{x}(0)=0, \quad V \in H^{3}(0,1) .
\end{gathered}
$$

The graph of $f(\cdot)-P_{0}$ depends on the value of $P_{0}$, i.e., the stress applied at the left of the material. In what follows and without loss of generality, we assume that the graph $f(\cdot)-P_{0}$ is of the form depicted in Fig. 2. Then the graph of $W(V)$ (see (4.2.1)) is given in Fig. 3. It is convenient to consider the problem (5.21) as the following overdetermined first-order system

$$
\begin{aligned}
& \lambda U^{\prime}(x)=f(V)-P_{0}, \quad V^{\prime}(x)=U(x), \\
& U(0)=0, \quad V(0)=V_{0}, \quad U(1)=0,
\end{aligned}
$$

where $V_{0}$ must be chosen in such a way that $U(1)=0$. The phase diagram for (5.22.1) is given in Fig. 4. It follows from this diagram that for a solution of (5.22) to exist, we must have $A<V_{0}<D$, or $V_{0} \in\{A, B\}$.

Multiplying (5.21.1) by $V_{x}$ and using (5.21.2), we see that

$$
\frac{-\lambda}{2} V_{x}^{2}+W(V)=W\left(V_{0}\right)
$$

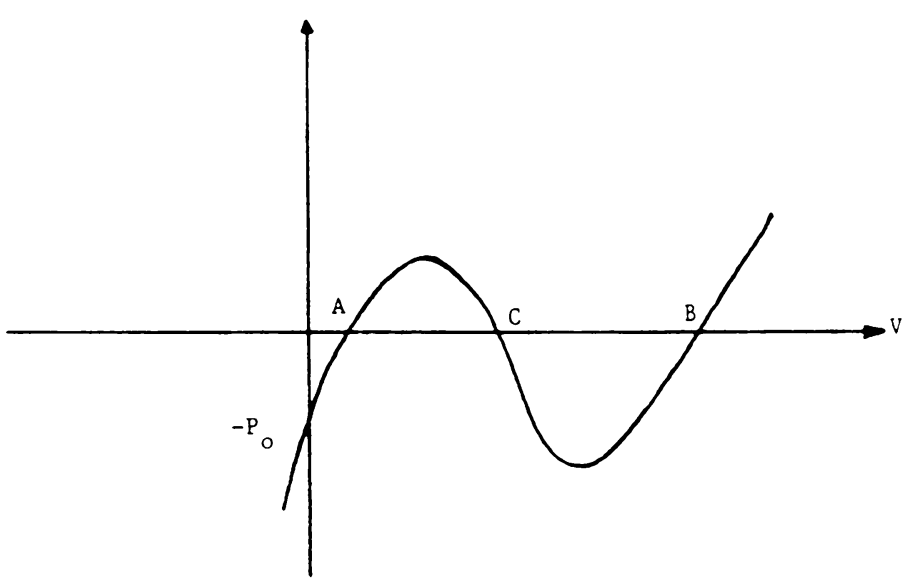

FIG. 2 


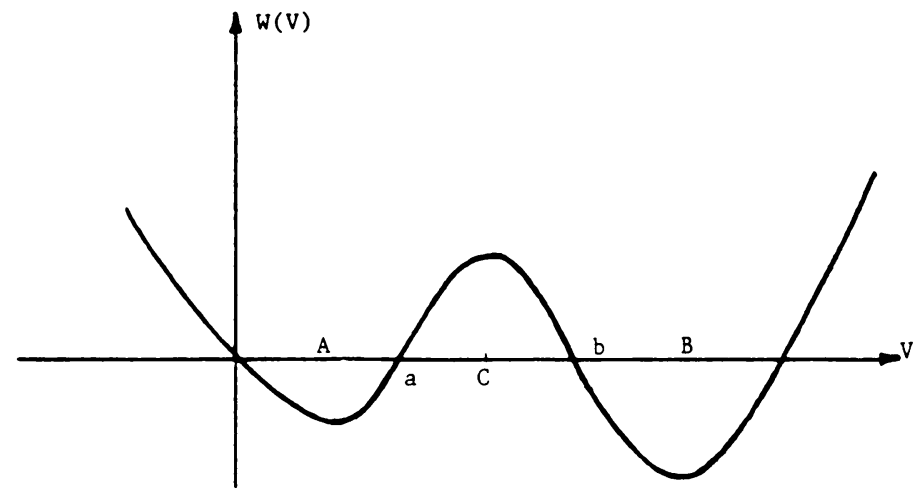

FIG. 3

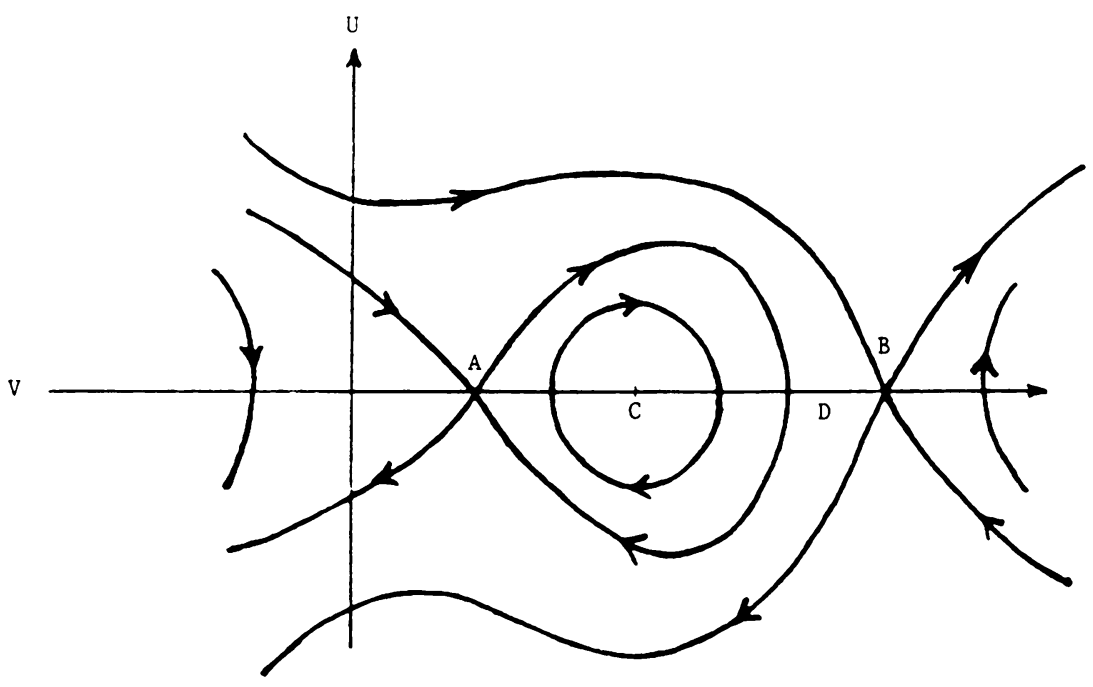

FIG. 4

Let $V_{1}\left(V_{0}\right)$ be the value of $V$ which occurs when the solution of $(5.22 .1)$ first crosses the $V$ axis. (We note from the phase diagram and (5.23) that $W\left(V_{1}\right)=$ $W\left(V_{0}\right)$.) Then (5.21.2) implies that for $V_{0}<C$,

$$
\sqrt{\lambda} \int_{V_{0}}^{V_{1}\left(V_{0}\right)} \frac{d V}{\sqrt{2\left(W(V)-W\left(V_{0}\right)\right)}}=\frac{1}{n}
$$

for some positive integer $n$. If $V_{0}>C$, then $V_{1}\left(V_{0}\right)<C$ and we need

$$
\sqrt{\lambda} \int_{V_{0}}^{V_{1}\left(V_{0}\right)} \frac{d V}{\sqrt{2\left(W(V)-W\left(V_{0}\right)\right)}}=-\frac{1}{n},
$$

for some positive integer $n$. By letting

$$
F\left(V_{0}\right)= \pm \int_{V_{0}}^{V_{1}\left(V_{0}\right)} \frac{d V}{\sqrt{2\left(W(V)-W\left(V_{0}\right)\right)}},
$$


depending on whether $V_{0}<C(+)$ or $V_{0}>C(-)$, we see that the solution of the initial value problem contained in (5.22) is a solution to (5.22) exactly when $F\left(V_{0}\right)=1 / n \sqrt{\lambda}$ for some positive integer $n$; or else $V_{0} \in\{A, B, C\}$, in which case we obtain a constant solution of (5.22).

It is routine to show that

$$
\lim _{V_{0}^{\prime} \rightarrow A^{+}} F\left(V_{0}\right)=\lim _{V_{0}^{\prime} \rightarrow D-} F\left(V_{0}\right)=\infty
$$

We assume that

$$
F(\cdot) \quad \text { is } C^{1} \text { on }(A, C) \cup(C, D)
$$

and note that it is a simple but tedious exercise to verify (5.28) under reasonable smoothness assumptions on $f$. We also observe that $W(A)<W(C)$ and so if $u_{0 x}=A, u_{0} \in E$, it follows that

$$
G\left(u_{0}, 0\right)<G(w, 0)
$$

where $w_{x}=C, w=C x-C$.

TheOREM 4. Let $W(A)<G(w, 0)-\varepsilon$ for some $\varepsilon>0$ and suppose that $\left(u_{0}, u_{1}\right) \in$ $B \times H$ satisfies

$$
G\left(u_{0}, u_{1}\right)<G(w, 0)-\varepsilon .
$$

Then there exists a set of measure zero, $S$, such that for $\lambda \notin S$ and $u$ the solution of (3.3), there exists $z \in Z$ with

$$
\lim _{t \rightarrow \infty}\|u(t)-z\|_{E}=0
$$

Proof. We begin with the following

Claim. Since $G\left(u_{0}, u_{1}\right)<G(w, 0)-\varepsilon$, there exists $r_{1}>0$ independent of $\left(u_{0}, u_{1}\right)$ in $B \times H$ satisfying (5.30) such that if $z \in \pi_{1}\left(\omega\left(u_{0}, u_{1}\right)\right)$, then $\left|z_{x}(0)-C\right|>$ $r_{1}$.

Proof of Claim. If the claim is false, there exist $\left(u_{0 n}, u_{1 n}\right) \in B \times H$ satisfying (5.30) and $z_{n} \in \pi_{1}\left(\omega\left(u_{0 n}, u_{1 n}\right)\right)$ such that $\left|z_{n x}(0)-C\right|<1 / n$. It follows that $z_{n} \rightarrow w$ in $E$ and so $G\left(z_{n}, 0\right) \rightarrow G(w, 0)$. By Lemma 7 and $(5.10), G\left(z_{n}, 0\right)<G(w, 0)-\varepsilon$; therefore $G(w, 0)<G(w, 0)-\varepsilon$, a contradiction. This proves the claim.

By Sard's theorem [13] and (5.28), the set of singular values of $F$ has measure zero and so there exists a set $S$ of measure zero such that $1 / n \sqrt{\lambda}$ is not a singular value of $F$ for all $n=1,2, \ldots$ wherever $\lambda \notin S$. For a fixed $\lambda \notin S$, (5.27) implies that for $z \in Z, \quad z_{x}(0) \in\{A, B\} \cup\left[A+r_{2}, D-r_{2}\right]$ where $r_{2}>0$ depends on $\lambda$. Therefore, for $z \in \pi_{1}\left(\omega\left(u_{0}, u_{1}\right)\right), z_{x}(0) \in\{A, B\} \cup[A+r, C-r] \cup[C+r, D-r]$ where $r=\min \left(r_{1}, r_{2}\right)$. Since $F$ is bounded away from zero on $[A+r, C-r] \cup[C+r, D-r]$, only finitely many values of $n$ can have the property that $1 / n \sqrt{\lambda}$ is a value of $F$ for $\left.z_{x}(0) \in[A+r, C-r)\right] \cup[C+r, D-r]$. Since $\lambda \notin S$ and $1 / n \sqrt{\lambda}$ is a regular value of $F$, it follows that for a fixed $y$ there are only finitely many elements of $F^{-1}(1 / n \sqrt{\lambda})$ in $\left.[A+r, C-r)\right] \cup[C+r, D-r]$. Therefore $\pi_{1}\left(\omega\left(u_{0}, u_{1}\right)\right)$ consists of finitely many points and consequently $u(t)$ converges in $E$ and $\pi_{1}\left(\omega\left(u_{0}, u_{1}\right)\right)$ consists of a single point. 


\section{REFERENCES}

[1] V. Alexiades and E. C. Aifantis, On the thermodynamic theory of fluid interfaces, infinite intervals, equilibrium solutions and minimizers, Mechanics of Microstructures, Report No. 9, 1984

[2] E. C. Aifantis and J. B. Serrin, The mechanical theory of fluid interfaces and Maxwell's rule, J. Coll. Int. Sci. 96, 517-529 (1983)

[3] G. Andrews and J. M. Ball, Asymptotic behavior and changes of phase in one-dimensional nonlinear viscoelasticity, J. Differential Equations 44, 306-341 (1982)

[4] J. M. Ball, On the asymptotic behavior of generalized processes with applications to nonlinear evolution equations, J. Differential Equations 27, 224-265 (1978)

[5] J. Carr, M. E. Gurtin, and M. Slemrod, One-dimensional structured phase transformations under prescribed loads, MRC Technical Summary Report \#2559, 1983

[6] C. M. Dafermos, Contraction semigroups and trend to equilibrium in continuum mechanics, Lecture Notes in Math. 503, 295-306 (1976)

[7] J. K. Hale, Dynamical systems and stability, J. Math. Anal. Appl., 26, 39-59 (1969)

[8] D. Henry, Geometric Theory of Semi linear Parabolic Equations, Lecture Notes in Math. Vol. 840, Springer, New York, 1981

[9] K. L. Kuttler, Regularity of weak solutions of some nonlinear conservation laws, Applicable Analysis, Vol. 26, Oct. 1987

[10] K. L. Kuttler and D. L. Hicks, Weak solutions of initial-boundary value problems for a class of nonlinear viscoelastic equations, Appl. Anal. (1) 26, 33-43 (1987)

[11] K. L. Kuttler and D. L. Hicks, Initial boundary value problems for the equation $u_{t t}=\left(\alpha\left(u_{x}\right) u_{x t}+\right.$ $\left.\sigma\left(u_{x}\right)\right)_{x}+f$, Quart. Appl. Math. (3) 46, 393-407 (1988)

[12] R. L. Pego, Phase transitions in one dimensional nonlinear viscoelasticity: admissibility and stability, Arch. Rational Mech. Anal. (4) 97, 353-394 (1987)

[13] M. Spivak, Calculus on Manifolds, W. A. Benjamin, 1965 